\title{
Effects of multiaxial cyclic loading conditions on the evolution of porous defects
}

\author{
Armel Mbiakop ${ }^{\mathrm{a}}$, Andrei Constantinescu and Kostas Danas \\ Laboratoire de Mécanique des Solides, CNRS, UMR 7649, Ecole Polytechnique, 91128 Palaiseau, \\ France
}

\begin{abstract}
Multiaxial loading conditions are one of the important parameters in estimating the lifetime of structure both in high and low cycle fatigue ([1-3]). In order to understand the coupling between the macroscopic multiaxial loading and the microscopic defects, we propose to investigate the evolution of an elasto-plastic porous material up to failure under low cycle fatigue conditions. The analysis is performed numerically, using finite elements, on a periodic 3D unit-cell under the assumption of finite strains and subjected to various stress triaxialities, translated as ratios between deviatoric, hydrostatic stress and Lode angles. The present discussion introduces several novel factors in the analysis: (i) 3D geometry in cyclic loading (ii) finite strains (iii) free evolving void shape (iiii) different hardening laws. That one of the important factors is the void shape and that its evolution during cyclic loading depends on its multiaxiality. Moreover, these factors will equally influence the apparent macroscopic hardening or softening of the material and the initiation of localized shear zones at the microscopic level. The Lode angle has a significant impact on the evolution of the aspect ratios and the ellipsoidicity of the pores, but has only a weak influence on the evolution of macroscopic variables such as the stress or the porosity. As a consequence, the results show that multiaxiality of the loading have an important on the evolution and growth of defects, pores in the present case problem, but are less important in the definition of the yield surface.
\end{abstract}

\section{Problem setting}

The scope of the present study is to investigate the effect of cyclic loading conditions and finite deformations upon microstructure evolution and material softening/hardening using finite element (FEM) periodic unit-cell calculations with 3D geometry at small and large number of cycles. The matrix material is described by isotropic $J 2$ plasticity considering the case, described previously, of the voids being much larger than the grain size but smaller than the specimen size. Nonetheless, the results obtained in the present study could still be valid, at least in a qualitative manner, in the case of materials with large, but not strongly anisotropic (e.g., FCC) grains comprising intragranular voids that are not of a nanometer size. In order to carry out this study, we define a periodic porous medium with cubic unit-cell geometry as well as the loading conditions used. The interest in this work is the analysis of a

\footnotetext{
${ }^{a}$ Corresponding author: mbiakop@lms . polytechnique.fr
}

This is an Open Access article distributed under the terms of the Creative Commons Attribution License 4.0, which permits unrestricted use, distribution, and reproduction in any medium, provided the original work is properly cited. 


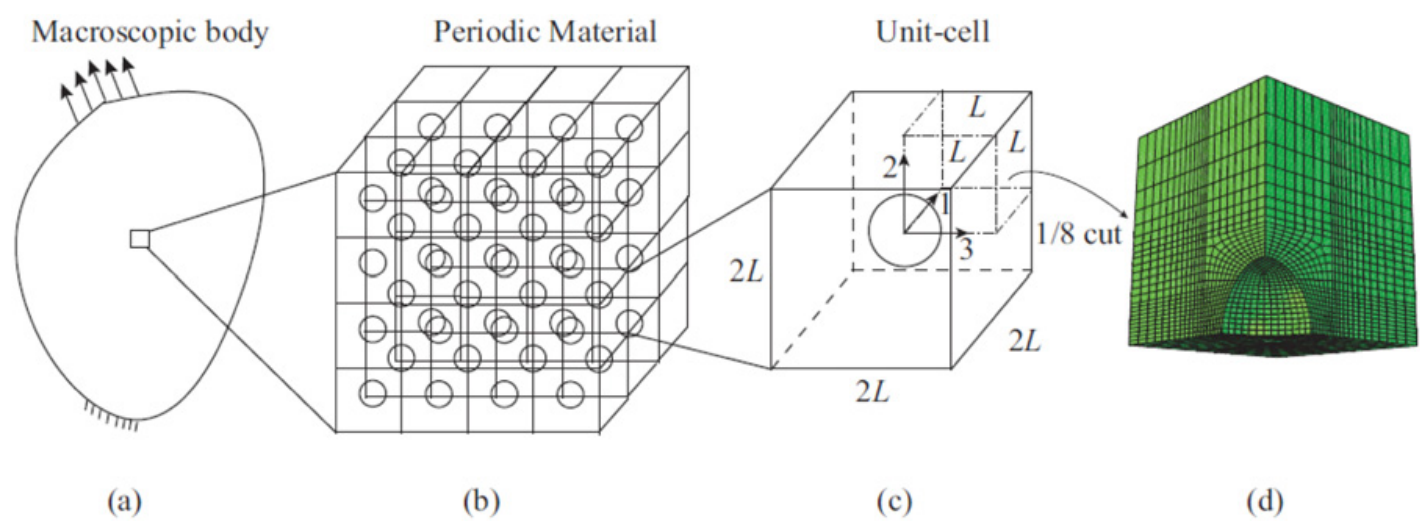

Figure 1. (a) A three-dimensional specimen at the macroscopic scale made-up of a periodic porous material. (b) A periodic porous material with cubic symmetry at the micron scale. (c) Geometry of a single cubic unit-cell with a void at the center and (d) mesh of the $1 / 8$ cut of the unit-cell geometry.
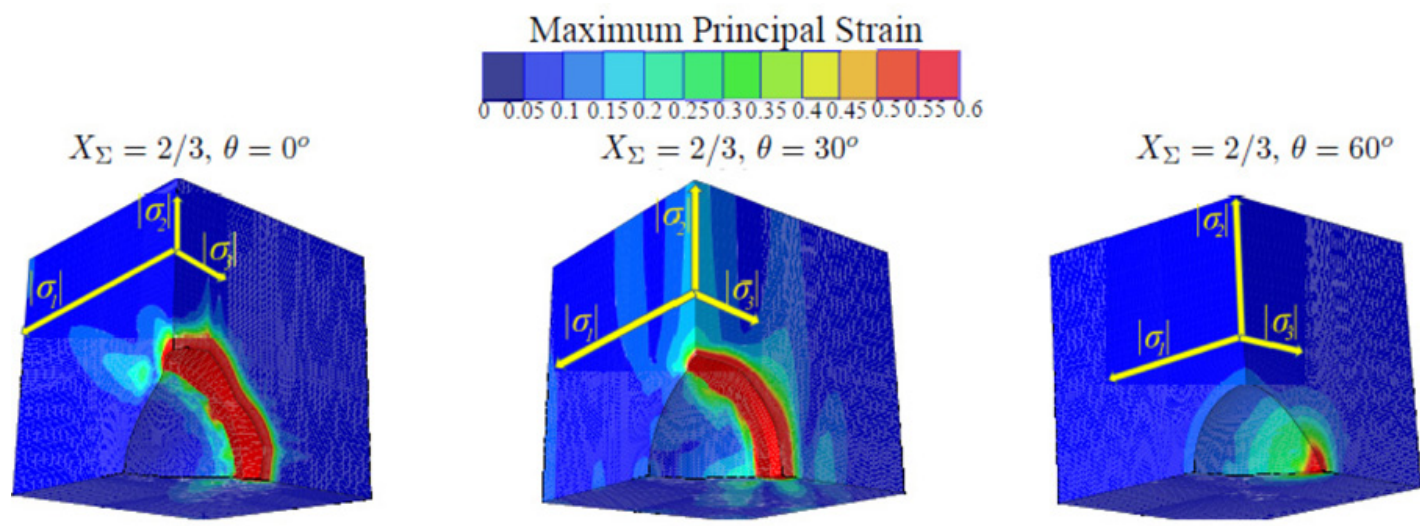

Figure 2. Contours of the maximum principal logarithmic strain at 40 cycles at triaxiality $2 / 3$ and for several Lode angle cases.

cubic periodic unit-cell comprising a microstructure of a spherical void under cyclic loading conditions subjected to a constant average stress triaxiality and average Lode angle loading state. A critical aspect of the present study is the use of a finite strain analysis contrary to the more common small strain analysis used when studying cyclic loading conditions. This will allow to track the evolution of the void geometry due to the local large strains at the reference configuration.

\section{Results and discussion}

In this section, we present some results obtained by the previously described loading conditions. The cyclic loading conditions are parametrized by the use of two different values of the triaxiality (2/3 and 3) and different values of Lode angle. At this point, we note that the evolution of the void shape does not describe adequately the deformation mechanisms near the void surface. In fact, for most of the computations presented here (except for the case of Lode angle $60^{\circ}$ ) significant localization of the deformation occurs at the surface of the void. To illustrate this, we show, in Fig. 2 contours of the deformed unit-cell at 40 cycles for Lode angles $=0,30,60^{\circ}$ and a stress triaxiality fo $2 / 3$. To emphasize 
further the relative magnitude of each of the stress components $\left|\sigma_{i}\right|(i=1,2,3)$, we explicitly show them at the top corner of the displayed unit-cell. In particular, we observe a strong localization of the deformation (strains exceeding 60\%) in a small zone of the void surface whose size depends upon the mesh size.

It is worth noting at this point that recent experiments by continuous X-ray tomography ([4]) have revealed the effect of stress triaxiality upon void shape and growth, albeit in monotonic loading conditions. As suggested by the present numerical calculations ([5]), the void shape changes under cyclic loadings are very different when compared to those obtained for monotonic loadings ([6]).

\section{References}

[1] V. Monchiet, E. Charkaluk, D. Kondo, Mechanics Research Communications 35, (2008)

[2] S. Tabibian, E. Charkaluk, A. Constantinescu, F. Szmytka, A. Oudin, Int. Journal of Fatigue (2012)

[3] R.M. McMeeking, C.L. Hom, Int. Journal of Fracture 42 (1990)

[4] A. Hosokawa, D.S. Wilkinson, J., Kang, E. Maire, Acta Materialia 60 (2012)

[5] A. Mbiakop, A. Constantinescu, K. Danas, Int. Jouranl of Plasticity (submitted), (2014)

[6] K. Danas, P. Ponte Castañeda, Eur. Journal of Mechanics A/Solids 28 (2009) 